PROCEEDINGS OF THE

AMERICAN MATHEMATICAL SOCIETY

Volume 133, Number 3, Pages 937-943

S 0002-9939(04)07684-1

Article electronically published on September 29, 2004

\title{
COUNTABLY COMPACT GROUPS FROM A SELECTIVE ULTRAFILTER
}

\author{
S. GARCIA-FERREIRA, A. H. TOMITA, AND S. WATSON
}

(Communicated by Alan Dow)

\begin{abstract}
We prove that the existence of a selective ultrafilter on $\omega$ implies the existence of a countably compact group without non-trivial convergent sequences all of whose powers are countably compact. Hence, by using a selective ultrafilter on $\omega$, it is possible to construct two countably compact groups without non-trivial convergent sequences whose product is not countably compact.
\end{abstract}

\section{INTRODUCTION}

The first example of a countably compact group without non-trivial convergent sequences was constructed, assuming $C H$, by A. Hajnal and I. Juhász [7]. A second example was discovered by E. K. van Douwen [3] under the assumption of $M A$, and one of the most recent examples lies in [10. All known examples of such a topological group use some form of $M A$. A similar situation holds in the problem of the existence, in $Z F C$, of two countably compact groups whose product is not countably compact (see, for instance, 3], 8], 9] and [10]). In this paper, we will construct two countably compact groups without non-trivial convergent sequences whose product is not countably compact from a selective ultrafilter. We also construct a countably compact group without non-trivial convergent sequences all of whose powers are countably compact from a selective ultrafilter on $\omega$.

We shall use standard notation. If $\left\{x_{\xi}: \xi<\mathfrak{c}\right\} \subseteq\{0,1\}^{\mathfrak{c}}$ and $F \in[\mathfrak{c}]^{<\omega}$, then $x_{F}=\sum_{\xi \in F} x_{\xi}$. The type of a point $p \in \beta(\omega) \backslash \omega=\omega^{*}$ is denoted by $T(p)=\left\{q \in \omega^{*}: \exists\right.$ a bijection $\left.f: \omega \rightarrow \omega(\bar{f}(p)=q)\right\}$, where $\bar{f}: \beta(\omega) \rightarrow \beta(\omega)$ denotes the Stone-Čech extension of $f$. An ultrafilter $p \in \omega^{*}$ is called selective if for every $f: \omega \rightarrow \omega$ there is $A \in p$ such that $\left.f\right|_{A}$ is either constant or one-to-one (the reader may find other combinatorial statements equivalent to selectivity in the book [2]).

The following concept has been very useful in the construction of countably compact spaces with certain properties.

Received by the editors March 3, 2003 and, in revised form, November 20, 2003.

2000 Mathematics Subject Classification. Primary 54G20, 54D80, 22A99; Secondary 54H11.

Key words and phrases. $p$-limit, $p$-compact, selective ultrafilter, countably compact group, topological group.

This research was supported by CONACYT grant no. 40057-F and DGAPA grant no. IN104601.

(C)2004 American Mathematical Society 
Definition 1.1 (A. R. Bernstein [1]). Let $p \in \omega^{*}$, and let $\left(x_{n}\right)_{n<\omega}$ be a sequence in a space $X$. We say that $x$ is a $p$-limit point of $\left(x_{n}\right)_{n<\omega}$, we write $x=p$ - $\lim _{n \rightarrow \omega} x_{n}$, if for every neighborhood $V$ of $x,\left\{n<\omega: x_{n} \in V\right\} \in p$.

It is not difficult to prove that a space $X$ is countably compact iff every sequence of points in $X$ has a $p$-limit point in $X$, for some $p \in \omega^{*}$. The following class of spaces was introduced by A. R. Bernstein [1].

Definition 1.2. Let $p \in \omega^{*}$. A space $X$ is said to be $p$-compact if for every sequence $\left(x_{n}\right)_{n<\omega}$ of points of $X$ there is $x \in X$ such that $x=p$ - $\lim _{n \rightarrow \omega} x_{n}$.

We know that $p$-compactness is preserved under arbitrary products, for each $p \in \omega^{*}$. Hence, we can find countably compact spaces that are not $p$-compact for any $p \in \omega^{*}$ (see [5]). J. Ginsburg and V. Saks [6] showed that all powers of a space $X$ are countably compact iff there is $p \in \omega^{*}$ such that $X$ is $p$-compact.

For $p \in \omega^{*}$, we shall use the properties of the ultrapower $\left([\mathfrak{c}]^{<\omega}\right)^{\omega} / p$ considered as a vector space over the field $\{0,1\}$ with the symmetric difference $A \Delta B=$ $(A \backslash B) \cup(B \backslash A)$ as addition. For $p \in \omega^{*}$, an element of the ultrapower $\left([\mathfrak{c}]^{<\omega}\right)^{\omega} / p$ will be denoted by $[f]_{p}$, where $f: \omega \rightarrow[\mathfrak{c}]^{<\omega}$ is a function. For $F \in[\mathfrak{c}]^{<\omega}$, the constant function whose domain is $\omega$ and takes only the value $F$ will be denoted by $\vec{F}$. If $\alpha<\mathfrak{c}$ is an ordinal, then $\{\vec{\alpha}\}$ will be denoted by $\vec{\alpha}$.

\section{The EXAMPLeS}

Our group $G$ will be generated by a linearly independent subset of $\{0,1\}^{\mathfrak{c}}$.

For every selective ultrafilter $p \in \omega^{*}$, it is evident that

$$
\left([\mathfrak{c}]^{<\omega}\right)^{\omega} / p=\left\{[f]_{p}: f \in\left([\mathfrak{c}]^{<\omega}\right)^{\omega} \text { is one-to-one }\right\} \cup\left\{[\vec{F}]_{\mathrm{p}}: \mathrm{F} \in[\mathfrak{c}]^{<\omega}\right\} .
$$

Lemma 2.1. Let $p \in \omega^{*}$ be selective. Then, there exists a family of one-to-one functions $\left\{f_{\xi}: \xi<\mathfrak{c}\right\} \subseteq\left([\mathfrak{c}]^{<\omega}\right)^{\omega}$ such that:

1) $\bigcup_{n<\omega} f_{\xi}(n) \subseteq \max \{\omega, \xi\}$, for every $\xi<\mathfrak{c}$.

2) $\left\{\left[f_{\xi}\right]_{p}: \xi<\mathfrak{c}\right\} \cup\left\{[\vec{\beta}]_{p}: \beta<\mathfrak{c}\right\}$ is a base for $\left([\mathfrak{c}]^{<\omega}\right)^{\omega} / p$.

3) For every one-to-one function $g \in\left([\mathfrak{c}]^{<\omega}\right)^{\omega}$, there are distinct $\zeta_{0}, \zeta_{1}<\mathfrak{c}$ and two increasing sequences of positive integers $\left(n_{k}^{0}\right)_{k<\omega}$ and $\left(n_{k}^{1}\right)_{k<\omega}$ such that $f_{\zeta_{i}}(k)=g\left(n_{k}^{i}\right)$, for every $k<\omega$ and $i \in\{0,1\}$.

Proof. Let $\left\{g_{\xi}: \xi<\mathfrak{c}\right\}$ be an enumeration of all one-to-one functions of $\left([\mathfrak{c}]^{<\omega}\right)^{\omega}$ in such a way that each element is listed two times, and $\bigcup_{n<\omega} g_{\xi}(n) \subseteq \max \{\omega, \xi\}$, for every $\xi<\mathfrak{c}$. We proceed by transfinite induction. Let $\alpha<\mathfrak{c}$ and suppose that, for each $\xi<\alpha$, we have defined a one-to-one function $f_{\xi}: \omega \rightarrow[\mathfrak{c}]^{<\omega}$ such that:

i) For every $m<\omega$ there is $n<\omega$ such that $f_{\xi}(m)=g_{\xi}(n)$, for every $\xi<\alpha$.

ii) $\left\{\left[f_{\zeta}\right]_{p}: \zeta<\xi\right\} \cup\left\{[\vec{\beta}]_{p}: \beta<\mathfrak{c}\right\}$ is linearly independent, for every $\xi<\alpha$.

iii) If $\left\{\left[f_{\zeta}\right]_{p}: \zeta<\xi\right\} \cup\left\{\left[g_{\xi}\right]_{p}\right\} \cup\left\{[\vec{\beta}]_{p}: \beta<\mathfrak{c}\right\}$ is linearly independent, then $f_{\xi}=g_{\xi}$, for every $\xi<\alpha$.

If $\left\{\left[f_{\xi}\right]_{p}: \xi<\alpha\right\} \cup\left\{\left[g_{\alpha}\right]_{p}\right\} \cup\left\{[\vec{\beta}]_{p}: \beta<\mathfrak{c}\right\}$ is linearly independent, then we define $f_{\alpha}=g_{\alpha}$. Let us assume that $\left\{\left[f_{\xi}\right]_{p}: \xi<\alpha\right\} \cup\left\{\left[g_{\alpha}\right]_{p}\right\} \cup\left\{[\vec{\beta}]_{p}: \beta<\mathfrak{c}\right\}$ is not linearly independent. Now, let $\left\{A_{\mu}: \mu<\mathfrak{c}\right\}$ be an almost disjoint family of infinite subsets of $\omega$. For each $\mu<\mathfrak{c}$, let $h_{\mu}: \omega \rightarrow A_{\mu}$ be a bijection. Then, we define $h_{\alpha, \mu}: \omega \rightarrow[\mathfrak{c}]^{<\omega}$ by $h_{\alpha, \mu}(n)=g_{\alpha}\left(h_{\mu}(n)\right)$, for each $n<\omega$. It is evident that $\left\{n<\omega: h_{\alpha, \mu}(n)=h_{\alpha, \nu}(n)\right\}$ is finite for $\mu<\nu<\mathfrak{c}$. Hence, $\left\{\left[h_{\alpha, \mu}\right]_{p}: \mu<\mathfrak{c}\right\}$ 
are pairwise distinct. So, we can find $\mu_{\alpha}<\mathfrak{c}$ such that $\left[h_{\alpha, \mu_{\alpha}}\right]_{p} \notin\left\langle\left\{\left[f_{\zeta}\right]_{p}: \zeta<\right.\right.$ $\left.\xi\} \cup\left\{[\vec{\beta}]_{p}: \beta<\max \{\omega, \alpha\}\right\}\right\rangle$. Put $f_{\alpha}=h_{\alpha, \mu_{\alpha}}$. Clearly, conditions $i$ ) and iii) are satisfied. We know that $\left\{\left[f_{\xi}\right]_{p}: \xi \leq \alpha\right\} \cup\left\{[\vec{\beta}]_{p}: \beta<\max \{\omega, \alpha\}\right\}$ is linearly independent. Since $\bigcup_{n<\omega} f_{\xi}(n) \subseteq \max \{\omega, \alpha\}$, for every $\xi \leq \alpha$, we also have that $\left\{\left[f_{\xi}\right]_{p}: \xi \leq \alpha\right\} \cup\left\{[\vec{\beta}]_{p}: \beta<\mathfrak{c}\right\}$ is linearly independent. This shows that condition ii) holds. We claim that the family $\left\{f_{\xi}: \xi<\mathfrak{c}\right\}$ satisfies all the conditions. Indeed, by the construction, $\left\{\left[f_{\xi}\right]_{p}: \xi<\mathfrak{c}\right\} \cup\left\{[\vec{\beta}]_{p}: \beta<\mathfrak{c}\right\}$ is a base for $\left([\mathfrak{c}]^{<\omega}\right)^{\omega} / p$. Let us prove that condition 3) is satisfied. For this, take $\zeta_{0}, \zeta_{1}<\mathfrak{c}$ so that $\xi_{0}<\xi_{1}$ and $g=g_{\zeta_{0}}=g_{\zeta_{1}}$. From condition $i$ ) we can find two increasing sequences of positive integers $\left(n_{k}^{0}\right)_{k<\omega}$ and $\left(n_{k}^{1}\right)_{k<\omega}$ such that $f_{\zeta_{i}}(k)=g\left(n_{k}^{i}\right)$, for every $k<\omega$ and $i \in\{0,1\}$.

In what follows, we fix a family $\left\{f_{\xi}: \xi<\mathfrak{c}\right\} \subseteq\left([\mathfrak{c}]^{<\omega}\right)^{\omega}$ satisfying the three properties stated in Lemma [2.1, and enumerate $[\mathfrak{c}]^{<\omega} \backslash\{\emptyset\}$ as $\left\{F_{\alpha}: \alpha<\mathfrak{c}\right\}$.

Lemma 2.2. Let $p \in \omega^{*}$ be selective. Suppose that for every $\alpha<\mathfrak{c}$ we have a non-trivial homomorphism $\Phi_{\alpha}:[\mathfrak{c}]^{<\omega} \rightarrow\{0,1\}$ such that

i) $\Phi_{\alpha}(\{\xi\})=p-\lim _{n \rightarrow \omega} \Phi_{\alpha}\left(f_{\xi}(n)\right)$, for every $\xi<\mathfrak{c}$; and

ii) $\Phi_{\alpha}\left(F_{\alpha}\right)=1$.

For $\xi<\mathfrak{c}$, we define $x_{\xi} \in\{0,1\}^{\mathfrak{c}}$ by $x_{\xi}(\alpha)=\Phi_{\alpha}(\{\xi\})$, for every $\alpha<\mathfrak{c}$. Then, the set $X=\left\{x_{\xi}: \xi<\mathfrak{c}\right\}$ is linearly independent in $\{0,1\}^{\mathfrak{c}}$ and $G=\langle X\rangle$ is a p-compact group without non-trivial convergent sequences.

Proof. Let $\left\{\xi_{0}, \ldots ., \xi_{k}\right\} \in[\mathfrak{c}]^{<\omega}$. Choose $\alpha<\mathfrak{c}$ such that $F_{\alpha}=\left\{\xi_{0}, \ldots ., \xi_{k}\right\}$. Then, by $i i)$,

$$
\left(x_{\xi_{0}}+\ldots+x_{\xi_{k}}\right)(\alpha)=\Phi_{\alpha}\left(\left\{\xi_{0}\right\}\right)+\ldots .+\Phi_{\alpha}\left(\left\{\xi_{k}\right\}\right)=\Phi_{\alpha}\left(F_{\alpha}\right)=1 .
$$

This shows that $\left\{x_{\xi}: \xi<\mathfrak{c}\right\}$ is linearly independent in $\{0,1\}^{\mathfrak{c}}$. Now we will show that $G$ is $p$-compact. Before proving this, notice from clause $i$ ) that

$$
x_{\xi}=p-\lim _{n \rightarrow \omega} \sum_{\mu \in f_{\xi}(n)} x_{\mu}=p-\lim _{n \rightarrow \omega} x_{f_{\xi}(n)},
$$

for every $\xi<\mathfrak{c}$. Let $\left(a_{n}\right)_{n<\omega}$ be a sequence in $G$. Choose $g \in\left([\mathfrak{c}]^{<\omega}\right)^{\omega}$ such that $a_{n}=x_{g(n)}$, for every $n<\omega$. Since $p$ is selective, there is $A \in p$ such that $\left.g\right|_{A}$ is either constant or one-to-one. If $\left.g\right|_{A}$ is constant, then there is $F \in[\mathfrak{c}]^{<\omega}$ such that $\left\{n<\omega: x_{g(n)}=x_{F}\right\} \in p$ and so $x_{F}=p$ - $\lim _{n \rightarrow \omega} x_{g(n)}$. Let us assume that there is a one-to-one function $h \in\left([\mathfrak{c}]^{<\omega}\right)^{\omega}$ such that $\left.h\right|_{A}=\left.g\right|_{A}$. Since $\left\{\left[f_{\xi}\right]_{p}\right.$ : $\xi<\mathfrak{c}\} \cup\left\{[\vec{\beta}]_{p}: \beta<\mathfrak{c}\right\}$ is a base for $\left([\mathfrak{c}]^{<\omega}\right)^{\omega} / p$, there are $\xi_{0}, \ldots, \xi_{k}<\mathfrak{c}$ and $E \in[\mathfrak{c}]^{<\omega}$ such that $[h]_{p}=\left(\Delta_{i \leq k}\left[f_{\xi_{i}}\right]_{p}\right) \Delta\left(\Delta_{\mu \in E}[\vec{\mu}]_{p}\right)$. Hence, we can find $B \in p$ such that $B \subseteq A$ and $h(n)=\left(\Delta_{i \leq k} f_{\xi_{i}}(n)\right) \Delta E$, for every $n \in B$. It then follows that $x_{h(n)}=\sum_{i \leq k} x_{f_{\xi_{i}}(n)}+x_{E}$, for all $n \in B$. So,

$$
\sum_{i \leq k} x_{\xi_{i}}+x_{E}=p-\lim _{n \rightarrow \omega} x_{h(n)} .
$$

This shows that $G$ is $p$-compact. Let $\left(y_{n}\right)_{n<\omega}$ be a non-trivial sequence in $G$, and assume that there is a one-to-one function $g \in\left([\mathfrak{c}]^{<\omega}\right)^{\omega}$ such that $y_{n}=x_{g(n)}$. By clause 3) of Lemma 2.1, there are distinct $\zeta_{0}, \zeta_{1}<\mathfrak{c}$ and two increasing sequences of positive integers $\left(n_{k}^{0}\right)_{k<\omega}$ and $\left(n_{k}^{1}\right)_{k<\omega}$ such that $f_{\zeta_{i}}(k)=g\left(n_{k}^{i}\right)$, for every $k<\omega$ and $i \in\{0,1\}$. Since $x_{\zeta_{i}}=p$ - $\lim _{k \rightarrow \omega} x_{f_{\zeta_{i}}(k)}=p$ - $\lim _{k \rightarrow \omega} x_{g\left(n_{k}^{i}\right)}=p$ - $\lim _{k \rightarrow \omega} y_{n_{k}^{i}}$, 
for each $i \in\{0,1\}$, we must have that $x_{\zeta_{0}}$ and $x_{\zeta_{1}}$ are both cluster points of $\left\{y_{n}: n<\omega\right\}$.

Lemma 2.3. Let $p \in \omega^{*}$ be selective. For every $E_{0} \in[\mathfrak{c}]^{<\omega} \backslash\{\emptyset\}$, there are $\left\{b_{i}: i<\omega\right\} \in p$ and $\left\{E_{i}: 0<i<\omega\right\} \subseteq[\mathfrak{c}]^{<\omega}$ such that

1) $\omega \subseteq \bigcup_{i<\omega} E_{i}$;

2) $E_{i} \cup\left[\bigcup_{\xi \in E_{i}} f_{\xi}\left(b_{i}\right)\right] \subseteq E_{i+1}$, for every $i<\omega$; and

3) $\left\{f_{\xi}\left(b_{i}\right): \xi \in E_{i}\right\} \cup\left\{\{\mu\}: \mu \in E_{i}\right\}$ is linearly independent, for every $i<\omega$.

Proof. Put $F_{0}=E_{0}$, and let $F_{n+1}=n \cup F_{n} \cup\left[\bigcup_{\xi \in F_{n}} \bigcup_{m \leq n} f_{\xi}(m)\right]$, for every $1 \leq n<\omega$. Since $\left\{\left[f_{\xi}\right]_{p}: \xi \in S\right\} \cup\left\{[\vec{\beta}]_{p}: \beta<\mathfrak{c}\right\}$ is linearly independent, we have that

$$
A_{n}=\left\{k<\omega:\left\{f_{\xi}(k): \xi \in F_{n}\right\} \cup\left\{\{\mu\}: \mu \in F_{n}\right\}\right.
$$

is linearly independent $\} \in p$,

for every $n<\omega$. By the selectivity of $p$, we can find $A=\left\{a_{n}: n<\omega\right\} \in p$ such that $m<a_{m}<a_{n}$ and $a_{n} \in A_{n}$, for every $m<n<\omega$. Let us define a coloring $P_{0}$ and $P_{1}$ on $[\omega]^{2}$ as $\{a, b\} \in P_{0}$ iff $a<b, a, b \in A, a=a_{m}, b=a_{n}$ and $a_{m}<n$, and $\{a, b\} \in P_{1}$ otherwise. Since $p$ is selective, there is $B \in p$ such that $B \subseteq A$ and either $[B]^{2} \subseteq P_{0}$ or $[B]^{2} \subseteq P_{1}$. Choose $I \in[\omega]^{\omega}$ such that $B=\left\{a_{n}: n \in I\right\}$. Let $\left\{i_{k}: k<\omega\right\}$ be the enumeration of $I$ in increasing order. Suppose that $[B]^{2} \subseteq P_{1}$. Since $\left\{a_{i_{0}}, a_{i_{k}}\right\} \in P_{1}$, then $a_{i_{0}} \geq i_{k}$, for every $1 \leq k<\omega$, but this is a contradiction. Therefore, $[B]^{2} \subseteq P_{0}$. Hence, we have that $i_{k}<a_{i_{k}}<i_{k+1}$, for every $k<\omega$. By using this, we obtain that

$$
F_{i_{k}} \cup\left[\bigcup_{\xi \in F_{i_{k}}} f_{\xi}\left(a_{i_{k}}\right)\right] \subseteq F_{i_{k+1}} \cup\left[\bigcup_{\xi \in F_{i_{k}}} \bigcup_{m<i_{k+1}} f_{\xi}(m)\right] \subseteq F_{i_{k+1}},
$$

for all $k<\omega$. Notice that, for every $k<\omega$,

$$
\left\{f_{\xi}\left(a_{i_{k}}\right): \xi \in F_{i_{k}}\right\} \cup\left\{\{\mu\}: \mu \in F_{i_{k}}\right\}
$$

is linearly independent, since $a_{i_{k}} \in A_{i_{k}}$. Then, for every $1 \leq k<\omega$, we define $E_{k}=F_{i_{k}}$ and, for every $k<\omega$, we put $b_{k}=a_{i_{k}}$. It is evident that 2) and 3) are satisfied. We remark that $E_{0} \subseteq F_{i_{0}}$ and

$$
\omega \subseteq \bigcup_{n<\omega} F_{n}=\bigcup_{k<\omega} F_{i_{k}}=\bigcup_{k<\omega} E_{k}
$$

and $B=\left\{b_{k}: k<\omega\right\} \in p$

Example 2.4. If $p \in \omega^{*}$ is selective, then there is a $p$-compact subgroup of size $\mathfrak{c}$ without non-trivial convergent sequences.

Proof. According to Lemma 2.2, it suffices to construct, for each $\alpha<\mathfrak{c}$, a non-trivial homomorphism $\Phi_{\alpha}:[\mathfrak{c}]^{<\omega} \rightarrow\{0,1\}$ such that

i) $\Phi_{\alpha}(\{\xi\})=p$ - $\lim _{n \rightarrow \omega} \Phi_{\alpha}\left(f_{\xi}(n)\right)$, for every $\xi<\mathfrak{c}$; and

ii) $\Phi_{\alpha}\left(F_{\alpha}\right)=1$.

Fix $\alpha<\mathfrak{c}$. By applying Lemma 2.3 to $E_{0}=F_{\alpha}$, we get $\left\{b_{i}: i<\omega\right\} \in p$ and $\left\{E_{i}: 0<i<\omega\right\} \subseteq[\mathfrak{c}]^{<\omega}$ such that

1) $\omega \subseteq \bigcup_{i<\omega} E_{i}=: E$

2) $E_{i} \cup\left[\bigcup_{\xi \in E_{i}} f_{\xi}\left(b_{i}\right)\right] \subseteq E_{i+1}$, for every $i<\omega$; and

3) $\left\{f_{\xi}\left(b_{i}\right): \xi \in E_{i}\right\} \cup\left\{\{\mu\}: \mu \in E_{i}\right\}$ is linearly independent, for every $i<\omega$. 
Now, suppose that for $i<\omega$, we have defined $\Phi_{\alpha}$ on $\left[E_{i}\right]^{<\omega}$ so that $\Phi_{\alpha}\left(F_{\alpha}\right)=1$ and $\Phi_{\alpha}\left(f_{\xi}\left(b_{i}\right)\right)=\Phi_{\alpha}(\{\mu\})$, for every $\xi, \mu \in E_{i}$. Since $\left\{f_{\xi}\left(b_{i+1}\right): \xi \in E_{i+1}\right\} \cup\{\{\mu\}$ : $\left.\mu \in E_{i+1}\right\}$ is linearly independent, and $E_{i} \cup \bigcup_{\xi \in E_{i}} f_{\xi}\left(b_{i}\right) \subseteq E_{i+1}$, we may extend $\Phi_{\alpha}:\left[E_{i}\right]^{<\omega} \rightarrow\{0,1\}$ to a homomorphism from $\left[E_{i+1}\right]^{<\omega}$ to $\{0,1\}$ in such a way that $\Phi_{\alpha}\left(f_{\xi}\left(b_{i+1}\right)\right)=\Phi_{\alpha}(\{\xi\})$, for every $\xi \in E_{i+1}$. Thus, we have defined $\Phi_{\alpha}$ on $[E]^{<\omega}$. Observe that $\Phi_{\alpha}\left(f_{\xi}\left(b_{i}\right)\right)=\Phi_{\alpha}(\{\xi\})$, for every $\xi \in E_{i}$ and $i<\omega$. Hence, $\left\{n<\omega: \Phi_{\alpha}\left(f_{\xi}(n)\right)=\Phi_{\alpha}(\{\xi\})\right\} \in p$, for every $\xi \in E$. Our next task is to extend $\Phi_{\alpha}$ to $[\mathfrak{c}]<\omega$. We will do this by transfinite induction on $\mathfrak{c} \backslash E$. Let $\gamma \in \mathfrak{c} \backslash E$ and suppose that $\Phi_{\alpha}$ has been defined on $[E \cup \gamma]^{<\omega}$. Since $f_{\gamma}(n) \subseteq \gamma$, for every $n<\omega$, $\Phi_{\alpha}(\{\mu\})$ has been defined for each $\mu<\gamma$ and $\{\{\gamma\}\} \cup\{\{\mu\}: \mu<\gamma\}$ is linearly independent, $\Phi_{\alpha}$ can be extended to $[E \cup(\gamma+1)]^{<\omega}$ in such a way that

$$
\Phi_{\alpha}(\{\gamma\})=p-\lim _{n \rightarrow \omega} \Phi_{\alpha}\left(f_{\gamma}(n)\right) .
$$

It is evident that $\Phi_{\alpha}$ satisfies the required properties.

The following example follows from E. K. van Douwen's construction [3, 6.1] applied to Example 2.4.

Example 2.5. If there is a selective ultrafilter on $\omega$, then there are two countably compact groups without non-trivial convergent sequences whose product is not countably compact.

\section{One MORE EXAMPLE}

In this section, we will improve a little bit Example 2.5

For $p \in \omega^{*}$, we say that a space is almost p-compact if for every sequence $\left(x_{n}\right)_{n<\omega}$ in $X$ there is a function $\sigma: \omega \rightarrow \omega$ such that $\bar{\sigma}(p) \in \omega^{*}$ and $\bar{\sigma}(p)-\lim _{n \rightarrow \omega} x_{n} \in X$ (this concept was introduced in [4]). It is evident that every $p$-compact space is almost $p$-compact, and every almost $p$-compact space is countably compact. All these notions are different from each other.

The following lemma is a generalization of Lemma 2.1.

Lemma 3.1. Let $p \in \omega^{*}$ be a selective ultrafilter. Then, there exists a family of one-to-one functions $\left\{f_{\xi}: \omega \leq \xi<\mathfrak{c}\right\} \subseteq\left([\mathfrak{c}]^{<\omega}\right)^{\omega}$ and pairwise disjoint sets $I_{0}, I_{1}, I_{2}, I_{3} \in[\mathfrak{c} \backslash \omega]^{\mathfrak{c}}$ such that:

a) $\bigcup_{n<\omega} f_{\xi}(n) \subseteq \xi$ for every $\omega \leq \xi<\mathfrak{c}$.

b) b.0) $\bigcup_{n<\omega} f_{\xi}(n) \subseteq \omega$, for every $\xi \in I_{0}$.

b.1) $\bigcup_{n<\omega} f_{\xi}(n) \subseteq \omega$, for every $\xi \in I_{1}$.

b.2) $\bigcup_{n<\omega} f_{\xi}(n) \subseteq I_{0} \cup I_{2}$, for every $\xi \in I_{2}$.

b.3) $\bigcup_{n<\omega} f_{\xi}(n) \subseteq I_{1} \cup I_{3}$, for every $\xi \in I_{3}$.

c) $\left\{\left[f_{\xi}\right]_{p}: \omega \leq \xi<\mathfrak{c}\right\} \cup\left\{[\vec{\beta}]_{p}: \beta<\mathfrak{c}\right\}$ is linearly independent.

d) For every $j \in\{0,1\}$ and for every one-to-one function $g \in\left([\omega]^{<\omega}\right)^{\omega}$, there exists a bijection $\sigma: \omega \rightarrow \omega$ and $\xi \in I_{j}$ such that $[g \circ \sigma]_{p}=\left[f_{\xi}\right]_{p}$.

e) For every $j \in\{0,1\},\left\{\left[f_{\xi}\right]_{p}: \xi \in I_{j+2}\right\} \cup\left\{[\vec{\beta}]_{p}: \beta \in I_{j} \cup I_{j+2}\right\}$ is a base for $\left(\left[I_{j} \cup I_{j+2}\right]^{<\omega}\right)^{\omega} / p$.

Proof. Let $I_{0}, I_{1}, I_{2}$ and $I_{3}$ be a partition of $\mathfrak{c} \backslash \omega$ in subsets of size $\mathfrak{c}$, and let $\left\{g_{\xi}: \omega \leq \xi<\mathfrak{c}\right\}$ be such that:

i) For each $j \in\{0,1\}$, we have that $\left\{g_{\xi}: \xi \in I_{j}\right\}$ is an enumeration of all one-to-one functions in $\left([\omega]^{<\omega}\right)^{\omega}$. 
ii) For each $j \in\{0,1\}$, we have that $\left\{g_{\xi}: \xi \in I_{j+2}\right\}$ is an enumeration of all one-to-one functions in $\left(\left[I_{j} \cup I_{j+2}\right]^{<\omega}\right)^{\omega}$ in such a way that $\bigcup_{n<\omega} g_{\xi}(n) \subseteq \xi$, for every $\xi \in I_{j+2}$.

By applying the proof of Lemma 2.1 to $\left\{g_{\xi}: \xi \in I_{j}\right\}$, for $j \in 2,3$, we get a set of one-to-one functions $\left\{f_{\xi}: \xi \in I_{j}\right\}$ satisfying a), b.2), b.3), and e). On the other hand, we apply the proof of Lemma 2.1 to $\left\{g_{\xi}: \xi \in I_{0} \cup I_{1}\right\}$ to obtain a family of one-to-one functions $\left\{f_{\xi}: \xi \in I_{j}\right\}$ satisfying b.0) and b.1). Furthermore, $\left\{\left[f_{\xi}\right]_{p}: \xi \in I_{0} \cup I_{1}\right\} \cup\left\{[\vec{n}]_{p}: n<\omega\right\}$ is linearly independent. Thus, condition c) also holds. Let us see how we get condition d). Following the notation of the proof of Lemma 2.1 at stage $\alpha<\mathfrak{c}$, we choose $\mu_{\alpha}<\mathfrak{c}$ such that $\left\{\left[h_{\alpha, \mu_{\alpha}}\right]_{p}: \alpha<\mathfrak{c}\right\} \cup\left\{\left[f_{\xi}\right]_{p}\right.$ : $\xi<\alpha\} \cup\left\{[\vec{\beta}]_{p}: \beta<\mathfrak{c}\right\}$ is linearly independent. We know that $h_{\mu_{\alpha}}: \omega \rightarrow A_{\mu_{\alpha}}$ is a bijection. Now, pick $B \subseteq A_{\mu_{\alpha}}$ such that $|B|=\left|A_{\mu_{\alpha}} \backslash B\right|=\omega$ and $h_{\mu_{\alpha}}^{-1}(B) \in p$. Choose a bijection $\sigma: \omega \rightarrow \omega$ for which $\sigma(n)=h_{\mu_{\alpha}}(n)$ for every $n \in h_{\mu_{\alpha}}^{-1}(B)$ and $\sigma\left[\omega \backslash h_{\mu_{\alpha}}^{-1}(B)\right]=\omega \backslash B$. So, we define $f_{\alpha}(n)=g_{\alpha}(\sigma(n))$, for every $n<\omega$. Then, we have that $f_{\alpha}=g_{\alpha}(\sigma(n))=g_{\alpha}\left(h_{\mu_{\alpha}}(n)\right)=h_{\alpha, \mu_{\alpha}}(n)$, for every $n \in h_{\mu_{\alpha}}^{-1}(B)$. Therefore, $\left[f_{\alpha}\right]_{p}=\left[g_{\alpha} \circ \sigma\right]_{p}=\left[h_{\alpha, \mu_{\alpha}}\right]_{p}$. This shows condition d).

In the next example, we fix a family $\left\{f_{\xi}: \omega \leq \xi<\mathfrak{c}\right\} \subseteq\left([\mathfrak{c}]^{<\omega}\right)^{\omega}$ and sets $I_{0}, I_{1}, I_{2}, I_{3} \in[\mathfrak{c} \backslash \omega]^{\mathfrak{c}}$ satisfying all the properties of Lemma 3.1 .

Example 3.2. If there is a selective ultrafilter on $\omega$, then there are two almost $p$-compact groups whose product is not countably compact.

Proof. By using clause c), Lemma 2.3 and the proof of Example 2.4, we can define, for every $\alpha<\mathfrak{c}$, a non-trivial homomorphism $\Phi_{\alpha}:[\mathfrak{c}]^{<\omega} \rightarrow\{0,1\}$ so that

i) $\Phi_{\alpha}(\{\xi\})=p$ - $\lim _{n \rightarrow \omega} \Phi_{\alpha}\left(f_{\xi}(n)\right)$, for every $\omega \leq \xi<\mathfrak{c}$; and

ii) $\Phi_{\alpha}\left(F_{\alpha}\right)=1$.

Hence, for each $\xi<\mathfrak{c}$ we define $x_{\xi}(\alpha)=\Phi_{\alpha}(\{\xi\})$, for every $\alpha<\mathfrak{c}$. Then, we have that $\left\{x_{\xi}: \xi<\mathfrak{c}\right\}$ is a linearly independent subset of $\{0,1\}^{\mathfrak{c}}$ and $x_{\xi}=$ $p$ - $\lim _{n \rightarrow \omega} x_{f_{\xi}(n)}$, for every $\omega \leq \xi<\mathfrak{c}$. We put

$E=\left\langle\left\{x_{n}: n<\omega\right\}\right\rangle$,

$H_{0}=\left\langle\left\{x_{\xi}: \xi \in I_{0} \cup I_{2}\right\}\right\rangle$,

$H_{1}=\left\langle\left\{x_{\xi}: \xi \in I_{1} \cup I_{3}\right\}\right\rangle$,

$G_{0}=E+H_{0}=\left\langle\left\{x_{\xi}: \xi \in \omega \cup I_{0} \cup I_{2}\right\}\right\rangle$ and

$G_{1}=E+H_{1}=\left\langle\left\{x_{\xi}: \xi \in \omega \cup I_{1} \cup I_{3}\right\}\right\rangle$.

It is evident that $G_{0} \cap G_{1}=E$. Hence, we deduce that $G_{0} \times G_{1}$ is not countably compact. As in Lemma2.2, both $H_{0}$ and $H_{1}$ are $p$-compact groups. We shall show that $G_{j}$ is almost $p$-compact, for $j \in\{0,1\}$. For this, fix a sequence $\left(a_{n}\right)_{n<\omega}$ in $G_{j}$. Choose two sequences $\left(e_{n}\right)_{n<\omega}$ in $E$ and $\left(h_{n}\right)_{n<\omega}$ in $H_{j}$ so that $a_{n}=e_{n}+h_{n}$, for every $n<\omega$. By the selectivity of $p$, there is $A \in p$ such that either $e_{n}=e$, for all $n \in A$, for some $e \in E$, or the function $n \rightarrow e_{n}$, for $n \in A$, is one-toone. In the former case, $e+h=p$ - $\lim _{n \rightarrow \omega}\left(e_{n}+h_{n}\right) \in E+H_{j}=G_{j}$, where $h=p$ - $\lim _{n \rightarrow \omega} h_{n}$. In the latter case, we can find a one-to-one function $g \in\left([\omega]^{<\omega}\right)^{\omega}$ such that $e_{n}=x_{g(n)}$, for every $n \in A$. According to clause e) of Lemma 3.1, there are a bijection $\sigma: \omega \rightarrow \omega$ and $\xi \in I_{j}$ such that $[g \circ \sigma]_{p}=\left[f_{\xi}\right]_{p}$. Pick $B \in p$ so that $B \subseteq A$ and $g(\sigma(n))=f_{\xi}(n)$, for every $n \in B$. Hence, $e_{\sigma(n)}=x_{g(\sigma(n))}=x_{f_{\xi}(n)}$, for every $n \in B$. This implies that

$$
p-\lim _{n \rightarrow \omega} e_{\sigma(n)}=p-\lim _{n \rightarrow \omega} x_{f_{\xi}(n)}=x_{\xi} \in H_{j} .
$$


So, $x_{\xi}=\bar{\sigma}(p)-\lim _{n \rightarrow \omega} e_{n}$ and $q=\bar{\sigma}(p) \in T(p)$. Since $H_{j}$ is $p$-compact, it is also $q$-compact. Thus, $q$ - $\lim _{n \rightarrow \omega} h_{n}=h \in H_{j}$. Hence, $q$ - $\lim _{n \rightarrow \omega} a_{n}=x_{\xi}+h \in$ $H_{j}+H_{j} \subseteq G_{j}$. Therefore, $G_{j}$ is almost $p$-compact.

Finally, we list some open problems that the authors were unable to solve.

Question 3.3. For an arbitrary $p \in \omega^{*}$, is there a $p$-compact group without nontrivial convergent sequences?

Question 3.4. Does the existence of a $P$-point in $\omega^{*}$ imply the existence of two countably compact groups whose product is not countably compact?

Question 3.5. Does the existence of a selective ultrafilter on $\omega$ imply the existence of a countably compact group whose square is not countably compact?

\section{ACKNOWLEDGMENT}

We are grateful to the referee for his helpful remarks and suggestions.

\section{REFERENCES}

1. A. R. Bernstein, A new kind of compactness for topological spaces, Fund. Math. 66 (1970), 185-193. MR0251697 (40:4924)

2. W. Comfort and S. Negrepontis, The Theory of Ultrafilters, Springer-Verlag, Berlin, 1974. MR0396267 (53:135)

3. E. K. van Douwen, The product of two countably compact topological groups, Trans. Amer. Math. Soc. 262 (1980), 417 - 427. MR0586725 (82b:22002)

4. S. Garcia-Ferreira, Quasi M-compact spaces, Czechoslovak Math. J. 46 (1996), 161 - 177. MR1371698 (97b:54033)

5. L. Gillman and M. Jerison, Rings of continuous functions, Lectures Notes in Mathematics No. 27, Springer-Verlag, 1976. MR0407579 (53:11352)

6. J. Ginsburg and V. Saks, Some applications of ultrafilters in topology, Pacific J. Math. 57 (1975), 403-418. MR0380736 (52:1633)

7. A. Hajnal and I. Juhász, A separable normal topological group need not be Lindelöf, Gen. Topology Appl. 6 (1976), 199-205. MR0431086 (55:4088)

8. K. P. Hart and J. van Mill, A countably compact topological group $H$ such that $H \times H$ is not countably compact, Trans. Amer. Math. Soc. 323 (1991), 811- 821. MR.0982236 (91e:54025)

9. A. H. Tomita, A group under $M A_{\text {countable }}$ whose square is countably compact but whose cube is not, Topology Appl. 91 (1999), 91-104. MR1664516 (2000d:54039)

10. A. H. Tomita, Countable compactness and finite powers of topological groups without convergent sequences, submitted.

Instituto de Matemáticas (UNAM), Apartado Postal 61-3, Xangari, 58089, Morelia, MichoACÁN, MÉXiCO

E-mail address: sgarcia@matmor.unam.mx

Departamento de Matemática, Instituto de Matemática e Estatística, Universidade de São Paulo, Caixa Postal 66281, CEP 05315-970, São Paulo, Brasil

E-mail address: tomita@ime.usp.br

Department of Mathematics, York University, 474700 Keele Street, M3P 1P3, Toronto, Ontario, Canada

E-mail address: watson@@mathstat.yorku.ca 